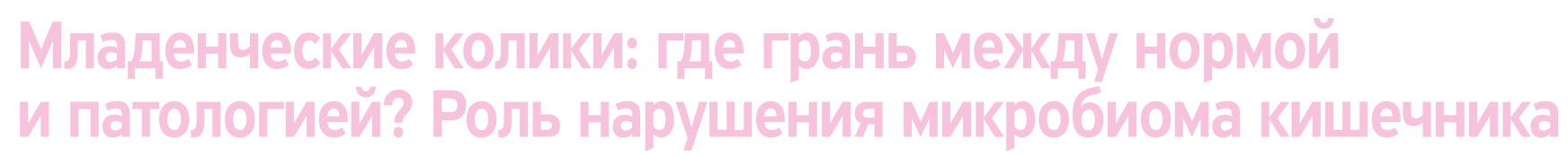

Л.Д. Панова, https://orcid.org/0000-0001-7570-7420, panov_home@ufacom.ru Башкирский государственный медицинский университет; 450008, Россия, Уфа, ул. Ленина, д. 3

\title{
Резюме
}

Младенческие колики относятся к наиболее часто встречающимся функциональным нарушениям желудочно-кишечного тракта у детей первого года жизни, ухудшающим состояние как самого ребенка, так и психологический климат в семье. Младенческая кишечная колика является мультифакториальным симптомокомплексом, требующим дифференциальной диагностики между нормой и патологией. До настоящего времени нет четкого понимания критериев необходимости диетической и медикаментозной коррекции данного состояния. В статье представлен анализ современной литературы по проблеме кишечных колик у детей первого года жизни. Патогенез функциональных кишечных колик определяется совокупностью причин, в т. ч. незрелостью центральной нервной системы, гипоталамо-гипофизарной системы, регуляцией иннервации кишечника и ферментативной системы, также выявлена определенная зависимость от психосоциальных факторов, большое влияние оказывает микрофлора кишечника. В 2014 г. H. Kianifar et al. в выводах по исследованию отметили, что мультипробиотик (семь штаммов пробиотиков и фруктоолигосахариды) значительно улучшил симптомы колик, в частности сокращение время плача (82,6\%), по сравнению с плацебо (35,7\%) на 7-й день приема ( $<$ 0,005). На 30-й день успех лечения составил 87 и $46 \%$ в группе синбиотиков и плацебо соответственно ( $<$ 0,01). Приведены результаты отечественного исследования мультипробиотика, проведенного И.Н. Захаровой и др. в 2016 г., которое показало, что после применения мультиштаммового пробиотика кишечные колики сохранились только у 3 (10\%) детей основной группы и у всех детей группы плацебо. После приема пробиотика у 80\% детей отмечена нормализация стула, а в группе плацебо нарушения стула (жидкий, водянистый стул или отсутствие стула с необходимостью опорожнения после клизмы) были зарегистрированы у 87\% детей. Показаны случаи из клинической практики с опытом применения мультиштаммового пробиотика при коликах.

Ключевые слова: функциональные нарушения желудочно-кишечного тракта, младенческие колики, микробиом кишечника, мультиштаммовый пробиотик

Для цитирования: Панова Л.Д. Младенческие колики: где грань между нормой и патологией? Роль нарушения микробиома кишечника. Медицинский совет. 2021;(17):246-254. https://doi.org/10.21518/2079-701X-2021-17-246-254.

Конфликт интересов: автор заявляет об отсутствии конфликта интересов.

\section{Infant colic: where is the line between norm and pathology, the role of disruption of the intestinal microbiome}

Luydmila D. Panova, https://orcid.org/0000-0001-7570-7420, panov_home@ufacom.ru

Bashkir State Medical University; 3, Lenin St., Ufa, 450008, Russia

\begin{abstract}
Infant colic is one of the most common functional disorders of the gastrointestinal tract in children of the first year of life, worsening the condition of both the child himself and the psychological climate in the family. Infant intestinal colic is a multifactorial symptom complex that requires differential diagnosis between norm and pathology. Until now, there is no clear understanding of the criteria for the need for dietary and drug correction of this condition. The article presents an analysis of the modern literature on the problem of intestinal colic in infants The pathogenesis of functional intestinal colic is determined by a combination of reasons, including the immaturity of the central nervous system, hypothalamic-pituitary system, regulation of the intestinal innervation and the enzymatic system, a certain dependence on psychosocial factors has also been identified, the intestinal microflora has a great influence. H. Kianifar et al. (2014) in the findings of a study noted that a multiprobiotic (seven strains of probiotics and fructooligosaccharides) significantly improved colic symptoms, in particular a reduction in crying time, (82.6\%) compared with placebo (35.7\%) on day 7 of intake ( $p<0.005)$. On day 30, treatment success was $87 \%$ and $46 \%$ in the synbiotics and placebo groups, respectively ( $p$ 0.01). The results of a domestic study by I.N. Zakharova et al. (2016) multiprobiotic, which showed that after the use of a multistrain probiotic, intestinal colic remained in $3(10 \%)$ children of the main group and in all children in the placebo group. After taking the probiotic, $80 \%$ of children showed normalization of stool, and in the placebo group, stool disorders (loose, watery stools or no stool with the need to empty after an enema) were recorded in $87 \%$ of children. Cases from clinical practice with experience of using a multistrain probiotic for colic are shown.
\end{abstract}

Keywords: functional disorders of the gastrointestinal tract, infant colic, intestinal microbiome, multi-strain probiotic

For citation: Panova L.D. Infant colic: where is the line between norm and pathology, the role of disruption of the intestinal microbiome. Meditsinskiy sovet = Medical Council. 2021;(17):246-254. (In Russ.) https://doi.org/10.21518/2079-

701X-2021-17-246-254.

Conflict of interest: the author declares no conflict of interest. 


\section{ВВЕДЕНИЕ}

Во всем мире крики ребенка первых месяцев жизни в связи с дисфункцией желудочно-кишечного тракта являются самой частой причиной обращения к педиатрам [1]. Термин «колика» происходит от греческого слова «коликос», что означает «боль в толстой кишке». Критерии определения младенческих колик менялись по мере изучения проблемы. Впервые детские колики были описаны у младенцев в 1954 г. M.A. Wessel et al. как «детский плач, продолжающийся 3 часа в день и более, по меньшей мере 3 дня в неделю, на протяжении не менее 3 недель» [2]. В дальнейшем данная нозология получила несколько другое определение: «эпизоды повышенной раздражительности, беспокойства или плача, возникающие и завершающиеся без очевидных причин, которые продолжаются 3 часа в день и более, по меньшей мере 3 дня в неделю, на протяжении не менее одной недели», что нашло свое отражение в Римских критериях III [3]. Римский консенсус IV предложил следующие критерии колик: симптоматика начинается и завершается у детей младше 5 мес.; повторный и пролонгированный период плача, беспокойства или раздражительности, которые возникают без очевидной причины и не могут быть предупреждены; нет признаков нарушения роста и развития, лихорадки или проявления иных болезней [4].

Младенческие кишечные колики встречаются, по данным различных авторов, от 5 до 80\% (в среднем у 20\%) детей на первом году жизни независимо от пола, вида вскармливания и являются мультифакториальным состоянием (табл.) [2, 5-8].

Приводятся многочисленные гипотезы механизма происхождения данного заболевания от погрешности в диете кормящей матери, нарушения техники кормления, пищевой непереносимости, аллергии к белкам коровьего молока до нарушения равновесия состава кишечной микрофлоры $[5,6,7,9,10]$. Патогенез функциональных кишечных колик определяется совокупностью причин, в т. ч. незрелостью центральной нервной системы, гипоталамогипофизарной системы, регуляцией иннервации кишечника и ферментативной системы, в результате которых возможны спазмы определенных отделов тонкого кишечника и повышенное газообразование [10-12]. Психосоциальные факторы: напряженное отношение в семье, беспокойство или депрессия у матери, нарушение взаимоотношений с ребенком, курение матери, а также особенности темперамента ребенка - являются факторами риска младенческих колик [5, 7, 13-15]. E.A. Cohen et al. предположили, что мелатонин, который поступает к младенцу с грудным молоком, может играть определенную роль в улучшении сна и уменьшении кишечных колик у детей на грудном вскармливании [16]. A. Burns и N. Thapar указывают, что разнообразные функции ЖКТ регулируются целым рядом систем, в т. ч. энтеральной нервной системой и кишечной микрофлорой [12]. S. Grenham et al., описывая ось «мозг - кишечник - кишечная микробиота», подчеркивают важность кишечной микробиоты для гомеостаза организма и регуляции функциональной активности кишечника [17]. У младенцев с коликами выявлено снижение уровня нормофло- ры, повышение количества кишечной палочки, клебсиеллы и другой условно-патогенной флоры [18-20]. В 2017 г. A. Pärtty et al., изучая биомаркеры сыворотки крови (цитокины, интерферон- $\gamma$, хемокины, моноцитарный хемотаксический макрофагальный воспалительный белок и др.), кишечный белок, связывающий жирные кислоты (биомаркер повреждения энтероцитов) и зонулин (биомаркер проницаемости кишечника), установили, что в дополнение к изменениям микробиоты кишечника колики у младенцев связаны с системным воспалением низкой степени тяжести, которое успешно лечится пробиотиками [21]. Повышенный рост протеолитической анаэробной микрофлоры сопровождается продукцией газов, обладающих потенциальной цитотоксичностью, что нередко приводит к повышению уровня воспалительного белка - кальпротектина и подтверждает роль воспаления слизистой оболочки кишечника в генезе колик [22]. В последних исследованиях, опубликованных в 2021 г., A. Loughman et al., используя метод секвенирования $16 \mathrm{~S}$ рРНК, показали, что несколько таксонов микробиоты, включая бифидобактерии, клостридии, лактобактерии и клебсиеллы, ассоциируются с тяжестью колик и базовый состав микробиоты может предсказать дальнейший плач через 4 нед. с точностью до 65\% [23].

Изучены не только ближайшие возможные последствия колик - это более раннее прекращение грудного вскармливания и «синдром встряхнутого ребенка» с большой вероятностью получения травм, но и такие серьезные отдаленные негативные последствия, как психологические

Таблица. Основные материнские и младенческие факторы риска колик

Table. Major maternal and infant risk factors for colic

\begin{tabular}{|c|c|}
\hline Материнские факторы риска & Младенческие факторы риска \\
\hline $\begin{array}{l}\text { Акушерские факторы: } \\
\text { - гестоз; } \\
\text { - кесарево сечение }\end{array}$ & $\begin{array}{l}\text { Нарушение количественного } \\
\text { и качественного состава } \\
\text { микробиома кишечника }\end{array}$ \\
\hline $\begin{array}{l}\text { Психосоциальные факторы: } \\
\text { - отсутствие социальной поддержки } \\
\text { во время беременности; } \\
\text { - возраст старше } 35 \text { лет; } \\
\text { - первый ребенок в семье; } \\
\text { - психоэмоциональная обстановка } \\
\text { в семье, конфликтные ситуации; } \\
\text { - матери-одиночки; } \\
\text { - курение, употребление алкоголя, } \\
\text { наркотиков во время беременности }\end{array}$ & $\begin{array}{l}\text { Морфофункциональная незрелость. } \\
\text { Ферментативная незрелость: } \\
\text { - транзиторная лактазная } \\
\text { недостаточность }\end{array}$ \\
\hline $\begin{array}{l}\text { Заболевания: } \\
\text { - метаболический синдром; } \\
\text { - мигрень }\end{array}$ & $\begin{array}{l}\text { Нейроэндокринные нарушения: } \\
\text { - недостаток мелатонина, } \\
\text { избыток серотонина; } \\
\text { - повышенный уровень мотилина } \\
\text { и грелина в крови }\end{array}$ \\
\hline \multirow[t]{3}{*}{$\begin{array}{l}\text { Ошибки ухода и вскармливания: } \\
\text { • нарушение диеты кормящей матери; } \\
\text { - нарушение техники вскармливания } \\
\text { (аэрофагия, перекорм и т. д.); } \\
\text { - неправильное разведение смеси }\end{array}$} & Гипоксическое поражение ЦНС \\
\hline & $\begin{array}{l}\text { Сенсибилизация к белку } \\
\text { коровьего молока }\end{array}$ \\
\hline & $\begin{array}{l}\text { Особенности темперамента } \\
\text { («холерики») }\end{array}$ \\
\hline
\end{tabular}


нарушения с агрессией и гиперактивностью [11, 24-27]. Выявлены взаимосвязи младенческих колик с рецидивирующей болью в животе, функциональными нарушениями желудочно-кишечного тракта, аллергическими заболеваниями, мигренью в последующем у детей школьного возраста [26, 28, 29]. E. Botha, K. Joronen, M. Kaunonen провели комплексный обзор литературы, опубликованной в период с января 2008 г.по апрель 2018 г., по базам данных MEDLINE, CINAHL, PsycINFO, Medic и Journals@Ovid (31 статья) по последствиям чрезмерного плача ребенка при коликах. Было сделано заключение, что чрезмерно плачущий ребенок в семье не только нарушает повседневную жизнь и ухудшает грудное вскармливание, но и разрушает семейные отношения, может привести к физическому и психическому истощению родителей, жестокому обращению. У самого младенца могут возникнуть различные проблемы со здоровьем в более позднем возрасте [30]. Результаты проведенного обзора литературы за 10 лет позволили авторам рекомендовать дальнейшие исследования для изучения основанных на фактических данных вмешательств, которые могут помочь при коликах младенцам и их семьям. Диагноз младенческих колик в большинстве случаев клинико-анамнестический. Основополагающим клиническим симптомом колики является чрезмерный и упорный громкий плач, преимущественно в вечерние часы, с выраженным беспокойством ребенка, покраснением лица, часто - урчанием в животе на фоне хорошего аппетита и нормальных показателей физического развития [4, 25]. Клиническая картина младенческих колик может скрывать такие заболевания, как гастроинтестинальная форма пищевой аллергии (чаще к белкам коровьего молока), нарушение толерантности к лактозе, гастроэзофагеальный рефлюкс, инфекция органов мочевой системы, перинатальное поражение ЦНС и др. [5-7, 9, 31-33]. Около 5-10\% случаев кишечных колик могут быть проявлением органических заболеваний, характеризуются упорным течением и наличием дополнительных «симптомов тревоги» (упорная диарея или запор, частые срыгивания или рвота с эпизодами кашля, кровь в кале, отказ от еды, нарушение весовых прибавок, замедление линейного роста, лихорадка, кривошея и др.), что требует проведения дифференциальной диагностики [25, 34].

Необходимость комплексного подхода в лечении младенческих колик обусловлено многофакторным характером данной патологии [35-37]. В некоторых случаях необходима терапия, направленная на то или другое звено патогенеза кишечных колик, ведущая роль которого установлена по результатам обследования. Первым шагом в коррекции младенческой колики можно считать психологическую поддержку и разъяснительную работу с родителями, которым необходимо объяснить, что колики являются преходящим состоянием и с возрастом уменьшатся $[1,26,35,36,38]$. Важным моментом в профилактике и коррекции колик, кроме физических методов, является диетотерапия [3, 8, 35, 36, 38]. При естественном вскармливании в свободном режиме необходимо исключить перекорм ребенка, из питания матери исключить продукты, повышающие газообразование в кишечнике, богатые экс- трактивными веществами, и цельное коровье молоко. При искусственном вскармливании рекомендуются адаптированная кисломолочная смесь и/или смеси, которые имеют в своем составе частично гидролизованный белок и обогащены пре-и/или пробиотиками (смеси серии «Комфорт»), при лактазной недостаточности - с пониженным содержанием лактозы [3, 8, 9, 35, 36, 38].

В литературе нет однозначных данных, указывающих на эффективность применения медикаментозной терапии при младенческих коликах, - оно носит индивидуальный характер. В ряде публикаций приводятся исследования по применению при коликах препаратов, содержащих симетикон, однако их рутинный прием не рекомендуется в связи с противоречивыми результатами [3, 35, 38].

В связи с тем, что существенную роль в патогенезе младенческих колик принадлежит нарушению становления микробиоценоза кишечника, с каждым годом возрастает интерес к роли определенных генетически сертифицированных пробиотических штаммов в поддержании функции микробиоты кишечника, в т. ч. у детей с коликами $[9,18-20$, 26, 36]. Y. Waikar подчеркивает, что недостаточно данных об эффективности некоторых штаммов пробиотиков и многообещающие результаты по ряду других [39]. Многочисленны исследования по применению при коликах у младенцев пробиотиков, содержащих Lactobacillus reuteri [19, $26,36,40]$. Канадское педиатрическое общество опубликовало результаты анализа систематических обзоров (SRs) и рандомизированных контролируемых исследований (РКИ) по эффективности пробиотиков при младенческих коликах, опубликованных в период с 1 января 2000 г. по 11 июля 2018 г. в базах MEDLINE, EMBASE, CINAHL, Cochrane, проведенного М. Karkhaneh et al. [40]. Пробиотик Lactobacillus reuteri использовался в большинстве (пяти из восьми) исследований для лечения колик и значительно снижал плач у детей, находящихся на грудном вскармливании, по сравнению с плацебо. Из шести исследований по профилактическому назначению пробиотика два показали значительное сокращение времени плача по сравнению с плацебо у детей на грудном вскармливании, еще два показали другие преимущества применения пробиотика, включая сокращение использования лекарств (симетикона) и визитов к врачу. Во включенных исследованиях не было выявлено никаких побочных явлений пробиотика Lactobacillus reuteri, он безопасен для здоровья детей [40]. Авторы считают, что применение определенных штаммов пробиотиков может быть вариантом управления коликами у младенцев. Результаты аналогичных обзоров и последующих исследований использования пробиотика Lactobacillus reuteri были созвучны с вышеизложенным обзором [41, 42]. Заслуживает внимание публикация иранских ученых о перспективе антенатальной профилактики колик пребиотиком: материнская пренатальная добавка с пробиотиком L. reuteri LR92 DSM 26866 в течение последних 4 нед. беременности может предотвратить возникновение и уменьшить тяжесть детской колики [43]. Периодически появляются данные об эффективности и других лактобацилл при коликах у младенцев: Lactobacillus acidophilus (NCFM) и Lactobacillus rhamnosus GG [44-46]. В 2016 г. группа польских ученых 
опубликовала данные РКИ и метаанализов, где звучит, что Lactobacillus rhamnosus GG является наиболее изученным бактериальным пробиотиком с доказанной эффективностью при различных заболеваниях и функциональных расстройствах желудочно-кишечного тракта, в т. ч. и детских коликах [45]. F. Savino et al. провели рандомизированное контролируемое исследование по оценке эффективности Lactobacillus rhamnosus ATCC 53103 вместе с отказом матери от коровьего молока при лечении детских колик. После приема в течение 28 дней добавки Lactobacillus rhamnosus младенцами с коликами медиана ежедневного плача достоверно снижалась (104 против 242 мин, p < 0,001), что сопровождалось значительным снижением кальпротектина в кале $(p=0,026)$ и увеличением количества лактобактерий ( $p=0,048)$ [46]. Для подтверждения этих результатов требуется двойное слепое плацебо-контролируемое исследование на более крупной когорте.

В последние годы приводятся данные об эффективности различных штаммов Bifidobacterium [47-49]. Рандомизированное двойное слепое плацебо-контролируемое исследование терапевтической эффективности Bifidobacterium animalis subsp. lactis BB- $12^{\circledR}$ при детских коликах показало уменьшение средней продолжительности ежедневного плача более чем на 50\% начиная со 2-й нед. приема BВ-12. В группе ВВ-12 наблюдалось увеличение численности бифидобактерий (со значительной корреляцией с сокращением времени плача), уровня бутирата и бета-дефензина-2, кателицидина, секреторного $\operatorname{lgA}$ и кальпротектина в кале [48]. Многоцентровое рандомизированное открытое параллельное контролируемое исследование продолжительностью 28 дней было проведено J.A. Maldonado-Lobón у 150 младенцев с коликами с применением B. breve СЕСТ7263. Установлено снижение ежедневного времени плача к концу первой недели и улучшение ночного сна [49]. В заключение было отмечено, что пробиотический штамм B. breve CЕСТ7263, выделенный из грудного молока, является безопасным и эффективным средством для лечения детских колик, оказывающим более раннее и более стойкое действие, чем эталонный назначенный препарат симетикон [49]. K. Chen et al. демонстрируют улучшение симптомов детской колики при пероральном введении смеси Bifidobacterium longum CECT7894 и Pediococcus pentosaceus CECT8330 [50].

Все более популярными в педиатрической практике становятся мультиштаммовые синбиотические препараты, поддерживающие многообразие кишечной микробиоты, нормализующие метаболическую активность и функциональную деятельность желудочно-кишечного тракта [51-57].

Одним из синбиотических мультиштаммовых препаратов, применяемых для коррекции колик у младенцев, являетсяБак-Сет ${ }^{\circledR}$ Беби (ADM Protexin,Великобритания) [51, 52, 55-57]. В его составе 7 генетически сертифицированных, кислотоустойчивых штаммов пробиотических культур, включенных в перечень европейского качества, имеющих оригинальное происхождение и обеспечивающих синергизм действия (Lactobacillus casei, L. rhamnosus, L. Acidophilus, Bifidobacterium infantis, B. longum, B. breve; термофильный стрептококк Streptococcus thermophilus) не менее 1 млрд микроорганизмов $\left(1 \times 10^{9}\right)$ KOE в одном саше, и фруктоолигосахариды (ФОС) в качестве пребиотика. При выборе пробиотика немаловажное значение имеет физиологичность его штаммового состава. Бак-Сет содержит виды бифидобактерий, которые могут применяться у детей с первых месяцев жизни (Bifidobacterium breve PXN 25, B. infantis PXN 27, B. longum PXN 30).

Бак-Сет ${ }^{\circledR}$ Беби не содержит искусственных красителей, ароматизаторов и генетически модифицированных организмов. Особенности производства обеспечивают высокую жизнеспособность штаммов при длительном хранении вне холодильника. Препарат соответствует стандарту GMP (Good Manufacturing Practice - надлежащая производственная практика). Бак-Сет ${ }^{\circledR}$ Беби - первый на российском рынке мультивидовой и мультиштаммовый синбиотик, разрешенный к применению у детей с рождения. Бак-Сет ${ }^{\circledR}$ Беби уникальный английский мультипробиотик, который снижает плач у младенцев с коликами до 90\% уже через 7 дней приема, что подтверждено зарубежными и российскими клиническими исследованиями [51, 52, 56, 57]. H. Kianifar et al. в выводах по исследованию отметили, что данный синбиотик (семь штаммов пробиотиков и ФОС) значительно снизил проявления симптомов колик, в частности сокращение время плача - 82,6\%, по сравнению с плацебо $35,7 \%$ на 7-й день приема ( $<<0,005)$. На 30-й день успех лечения составил 87 и 46\% в группе синбиотика и плацебо соответственно ( $<$ < 0,01) (рис. 1) [52].

И.Н. Захарова и др. установили, что после применения мультиштаммового пробиотика кишечные колики сохранились у 3 (10\%) детей основной группы и у всех детей группы плацебо (рис. 2). После приема пробиотика у $80 \%$ детей отмечена нормализация стула, а в группе плацебо нарушения стула (жидкий, водянистый стул или отсутствие стула с необходимостью опорожнения после клизмы) были зарегистрированы у 87\% детей [56]. На фоне приема мультиштаммового пробиотика отмечена тенденция к восстановлению активности облигатной толстокишечной микрофлоры, снижению активности факультативной и остаточной анаэробной и аэробной микрофло-

Рисунок 1. Доля детей, достигших первичной точки эффективности при приеме синбиотика [52]

Figure 1. Percentage of children who achieved the primary efficacy point due to intake of synbiotic [52]

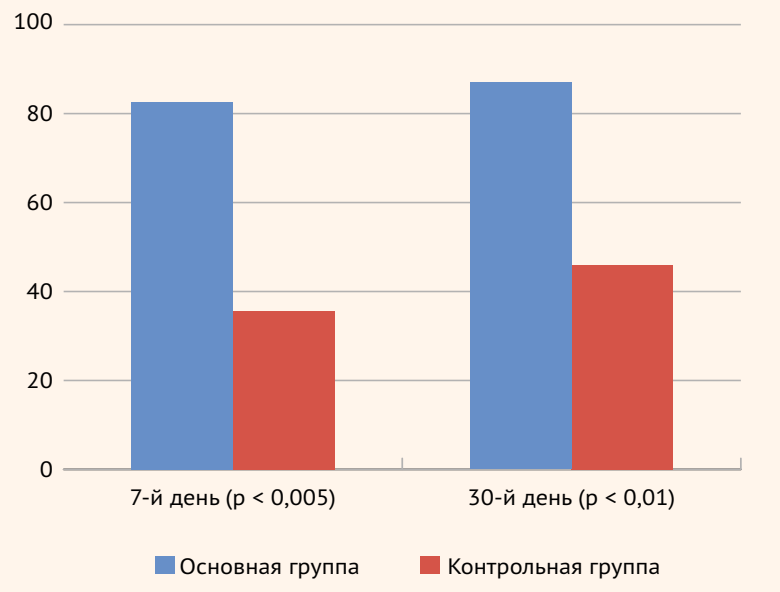


Рисунок 2. Динамика клинической симптоматики у детей основной и контрольной групп

Figure 2. Changes in clinical symptoms in children of the treatment and control groups

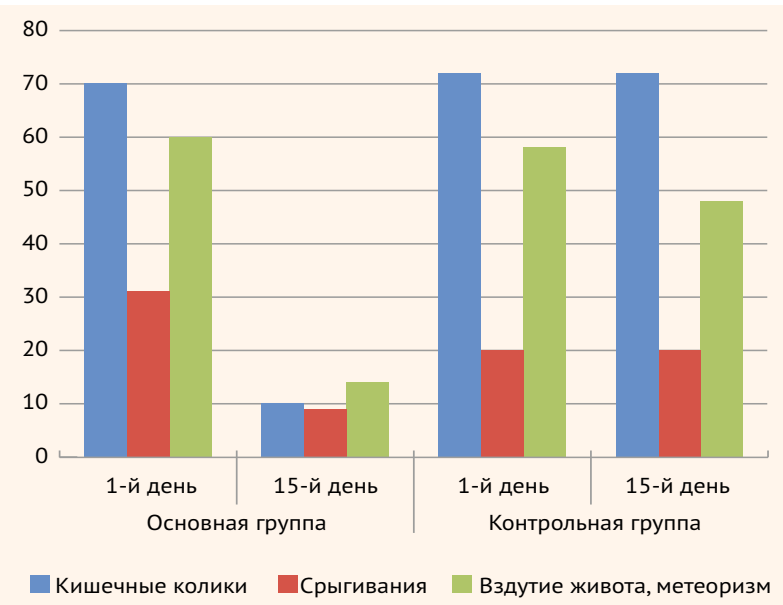

ры [56]. Авторы не столкнулись с осложнениями, связанными с применением синбиотика.

Таким образом, определенные штаммы пробиотиков могут играть решающую роль в манипулировании микробиотой. Введение пробиотиков, вероятно, поддерживает гомеостаз кишечника за счет модуляции проницаемости и перистальтики, влияя на ось «кишечник - мозг» и подавляя гиперчувствительность. Это решающая область в разработке профилактических и терапевтических стратегий при детских коликах [19]. Последний симптоматический обзор 2021 г., включающий 20 статей (15 рандомизированных контролируемых исследований и 5 метаанализов), подчеркивает, что пробиотики могут быть безопасно рекомендованы, если родители желают назначить лечение своим детям с коликами [58].

Приводим два клинических случая, подтверждающих высокую эффективность мультиштаммового синбиотика Бак-Сет ${ }^{\circledR}$ Беби при лечении младенческих колик.

\section{Клинический случай 1}

Девочка 3 мес. Жалобы на выраженное беспокойство ребенка, периодически пронзительный крик во время или после кормления, больше в вечернее время, более 3 ч/сут.

Родилась от первой доношенной беременности у женщины 19 лет. Беременность протекала на фоне ожирения, анемии, вегетососудистой дистонии по гипертоническому типу. В родах слабость потуг, медродоусиление. Девочка родилась с массой тела 3200 г, длиной 52 см, без асфиксии. К груди приложена в родильном зале, находилась на совместном пребывании с мамой. Выписана домой на 4-й день жизни. Вскармливалась грудным молоком по требованию до 10 раз в сутки. Стул после каждого кормления кашицеобразный или разжижен. Прибавка в массе достаточная. В возрасте 21 дня девочка после вечернего кормления стала громко и пронзительно кричать, поджимать ножки к животу, успокаивалась после отхождения газов или стула. Приступы повторялись ежедневно с нарастанием частоты и длительности, вызывая обеспокоенность мамы. Мама не соблюдала диетические принципы питания во время кормления грудью: употребляла жирную и жареную пищу, сырые овощи; для стимуляции лактации пила много горячего молока и чай с молоком.

После консультации участковым педиатром мама старалась придерживаться рекомендованной диеты, давала фитопрепараты ветрогонного действия в течение двух недель с временным эффектом, боли в животе у девочки сохранялись. Стул до 5 раз в сутки, кашицеобразный.

При первичном осмотре: масса тела 4900 г, рост 55 см, температура $36,9{ }^{\circ} \mathrm{C}$. Состояние удовлетворительное. Аппетит не нарушен. Кожные покровы розовые, чистые, суховаты. Видимая слизистая полости рта чистая, влажная, язык чистый. Дыхание в легких пуэрильное, хрипов нет. Тоны сердца чистые, ритмичные. ЧСС 110 уд/мин. Во время осмотра по требованию мама кормит ребенка грудью. К концу кормления отмечается покраснение лица, девочка начинает пронзительно кричать, резко поджимает ноги к животу. Живот вздут, умеренно напряжен, доступен пальпации, перкуторно - коробочный звук. Печень +0,5 см из-под края реберной дуги, селезенка не пальпируется. Стул кашицеобразный, без патологических примесей.

Результаты обследования: общий анализ крови, биохимический анализ крови: без особенностей. Копрология: в небольшом количестве нейтральный жир и йодофильная флора. Кальпротектин в кале: 91 мг/кг (норма до 6 мес. менее 538 мг/кг). Углеводы в кале: 0,1\% (норма 0-0,25\%). Анализ микробиоты тонкого кишечника методом хроматомасс-спектрометрии (XМС): нарушения микробиологического состава со снижением нормофлоры (лакто- и бифидобактерий), увеличения резидентных анаэробных микроорганизмов - пропионобактерий, клостридий тетани. Общая бактериальная нагрузка составила 20895 микроорганизмов (норма до 19 660). Плазминоген 33,71 мкг/мл (норма до 50 мкг/мл). Эндотоксин 0,025 наномоль/мл (норма до 0,5 наномоль/мл). УЗИ органов брюшной полости: повышенное газообразование. На основании анамнеза и результатов осмотра и обследования выставлен диагноз «R 10.4 «Кишечная колика».

Рекомендации: с целью снятия психологического напряжения в семье с родителями проведена беседа о причине колик как функциональном расстройстве желудочно-кишечного тракта у ребенка, которые постепенно будут угасать. Маме даны рекомендации по исключению из питания продуктов, которые вызывают метеоризм (огурцы, виноград, фасоль, кукуруза, майонез, молоко). Раскрыты правила постуральной терапии с наклоном ребенка животиком вниз в течение 10 мин для отхождения воздуха в результате аэрофагии. Рекомендован тактильный контакт кожи живота ребенка с кожей живота матери, выкладывание животиком на теплую пеленку. При выраженном метеоризме - использование газоотводной трубки и препаратов симетикона.

На фоне симптоматической терапии (симетикон без эффекта) в комплексную коррекцию колик включен мультиштаммовый синбиотик Бак-Сет ${ }^{\circledR}$ Беби согласно традиционной схеме, рекомендованной в инструкции к препарату, по 1 саше 1 раз в день перед кормлением курсом 14 дней. Клиническая эффективность Бак-Сет ${ }^{\circledR}$ Беби 
начала проявляться с 5-х сут. применения и заключалась в уменьшении выраженности и продолжительности болевого синдрома. В семье снизилась тревожность, появилась уверенность в благоприятном исходе колик. Спокойный психологический климат в окружении ребенка, снижение явлений метеоризма на фоне продолжения приема Бак-Сет® Беби значительно улучшили самочувствие ребенка и привели к полному купированию болевого синдрома к окончанию курса терапии.

\section{Клинический случай 2}

Пациент 3 мес. Жалобы на умеренные срыгивания после каждого кормления, вздутие живота, отсутствие стула до 3 сут. Выраженное беспокойство ребенка, периодически пронзительный крик во время кормления смесью, больше во второй половине дня.

Девочка от первой беременности у женщины 40 лет, роды путем операции кесарева сечения по поводу преждевременной отслойки плаценты при сроке 40 нед. Наследственность по аллергическим заболеваниям не отягощена. Беременность протекала на фоне ожирения, осложнилась гестозом, кольпитом. Девочка родилась с массой тела 4500 г, длиной 54 см, с асфиксией легкой степени. К груди приложена на 3-и сут. по состоянию мамы. Мама получала антибиотик в послеродовом периоде. Выписана из роддома на 8-й день жизни. Вскармливалась грудным молоком каждые 3 ч, однако в связи с гипогалактией у мамы с 1 мес. начала докармливаться стандартной смесью для доношенных детей на основе цельных белков коровьего молока «Нестожен». Девочка начала умеренно срыгивать после каждого кормления, отмечалось беспокойство, вздутие живота, крик при кормлении смесью. От переживаний у мамы молока практически не осталось, и с 2 мес. девочка полностью переведена на искусственное вскармливание. Усилилось газообразование, стул через сутки, часто поджимает ножки к животу, длительно кричит. После отхождения газов и стула самочувствие ребенка на некоторое время улучшалось. Стул, со слов мамы, с примесью слизи, иногда неоформленный.Прибавка в массе достаточная. Мама по рекомендации подруги начала кормить ребенка смесью на основе козьего молока «Нэнни», без ожидаемого эффекта.

Приступы колик повторялись ежедневно с нарастанием частоты и длительности во время и после кормления, вызывая обеспокоенность мамы. Нарушился сон, ребенок практически все время находился на руках. В семье нарушилась психологическая обстановка. Все внимание было приковано к состоянию ребенка, уходу за ним, попытке каким-либо образом его успокоить.

Данные первичного осмотра: масса тела 6950 г, рост 59 см, температура $36,6^{\circ} \mathrm{C}$. Состояние удовлетворительное. Несколько возбуждена. Психомоторное развитие соответствует возрасту. Кожные покровы розовые, щечки гиперемированы, суховаты. Слизистые чистые. Лимфатические узлы не увеличены. Отоскопия: отита не выявлено.Дыхание в легких пуэрильное, хрипов нет. Тоны сердца чистые, ритмичные. чСС 100 уд/мин. Живот мягкий, умеренно вздут, безболезненный при пальпации. Печень не увеличена, селезенка не пальпируется. Мочеиспускание не нарушено, безболезненно. Стул при осмотре кашицеобразный, неоформленный, желтый.

Результаты обследования: общий анализ крови, биохимический анализ крови: без особенностей. Копрология: $\mathrm{pH}$ 6,0, в небольшом количестве нейтральный жир, йодофильная флора, лейкоцитов до 5 в поле зрения. Кальпротектин в кале: 490 мг/кг (норма до 6 мес. менее 538 мг/кг). Углеводы в кале: 0,2\% (норма 0-0,25\%). Панкреатическая эластаза в кале: 300 мкг/г (норма более 200 мкг/г). Анализ микробиоты тонкого кишечника методом хромато-масс- спектрометрии (XМС): нарушения микробиологического равновесия со снижением нормофлоры (лакто- и бифидобактерий), увеличением резидентных аэробных и анаэробных микроорганизмов - пропионобактерий и актинобактерий. Общая бактериальная нагрузка составила 18656 микроорганизмов (норма до 19 660). Плазминоген 42,64 мкг/мл (норма до 50 мкг/мл). Эндотоксин 0,33 наномоль/мл (норма до 0,5 наномоль/мл). УЗИ органов брюшной полости: повышенное газообразование. На основании анамнеза и результатов осмотра и обследования выставлен диагноз «R 10.4 «Кишечная колика».

Рекомендации: с родителями проведена беседа о причине колик как о преходящем состоянии. Придана уверенность, что их ребенок здоров, требуется только правильный уход при появлении беспокойства: выкладывание на животик перед кормлением, массаж животика, постановка газоотводной трубки в случае задержки отхождения газиков и выраженном метеоризме. Кроме того, очень важен подбор смеси и нормализация колонизационной резистентности кишечника. Поскольку перинатальный анамнез отягощен факторами, нарушающими первичную микробную колонизацию желудочно-кишечного тракта новорожденного ребенка (кесарево сечение, гестоз, кольпит, искусственное вскармливания базовой смесью), обосновали необходимость приема пре- и пробиотиков. Подчеркнули, что эффект облегчения болей наступит не ранее чем через 2 нед. после смены питания и приема препаратов, нормализующих качественный состав микробиоты кишечника и поддерживающих ее многообразие. Рекомендован поэтапный переход в течение недели на смесь категории «Комфорт», например «Нутрилон Комфорт» на основе частичного гидролизата белков коровьего молока, обогащенную фрукто- и галактоолигосахаридами, аналогичными в грудном молоке, обладающими свойствами пребиотиков. Альтернативный вариант «Симилак Комфорт» с пребиотиком 2-фукозиллактоза. В комплексную коррекцию колик включен мультиштаммовый синбиотик Бак-Сет® Беби по 1 саше однократно со смесью курсом, рекомендуемым инструкцией к препарату, в течение 14 дней, что наряду с коррекцией питания способствовало улучшению самочувствия ребенка за счет уменьшения колик с 7-го дня и полного купирования болей к концу второй недели приема. Нивелировалась сухость кожи на щеках. Нормализовался сон. Стул ежедневно, кашицеобразный, без слизи. Семья обрела покой. Клиническая эффективность Бак-Сет ${ }^{\circledR}$ Беби не требовала повторного исследования микробиоты кишечника. 


\section{ЗАКЛЮЧЕНИЕ}

Анализ существующих научных данных демонстрирует, что патогенез младенческих колик продолжает оставаться предметом дискуссий и исследований, обосновывает необходимость персонализированного подхода к их коррекции. Проведенные многочисленные исследования показывают важность состояния кишечной микрофлоры в патогенезе колик, а полученные данные о возможности развития тяжелых ближайших и отдаленных последствий перенесенных в младенчестве колик для ребенка и членов семьи указывают на то, что кишечные колики нельзя оставлять без лечения. Представленный комплексный подход к ведению детей с младенческими коликами без значительного медикаментозного вмешательства и материальных затрат с применением мультиштаммового синбиотика Бак-Сет ${ }^{\circledR}$ Беби эффективен и обоснован. Исследования подтверждают эффективность Бак-Сета Беби в таких важных показателях, как снижение времени плача младенца и восстановление нормофлоры кишечника. Данные эффекты подтверждены клинической практикой применения мультиштаммового синбиотика.

Поступила / Received 13.09.2021

Поступила после рецензирования / Revised 30.09.2021 Принята в печать / Accepted 01.10.2021

\section{Список литературы}

1. Беляева И.А., Намазова-Баранова Л.С., Потехина Т.В. Младенческие колики - новый взгляд на старую проблему. Педиатрия. 2015;94(1):137-144. Режим доступа: https://pediatriajournal.ru/files/ upload/mags/344/2015_1_4212.pdf.

2. Wessel M.A., Cobb J.C., Jackson E.B., Harris G.S. Jr., Detwiler A.C. Paroxysmal fussing in infancy, sometimes called colic. Pediatrics. 1954;14(5):421-435. Available at: https://pubmed.ncbi.nlm.nih.gov/13214956.

3. Vandenplas Y., Gutierrez-Castrellon P., Velasco-Benitez C., Palacios J., Jaen D., Ribeiro $\mathrm{H}$. et al. Practical algorithms for managing common gastrointestinal symptoms in infants. Nutrition. 2013;29(1):184-194. https://doi. org/10.1016/j.nut.2012.08.008.

4. Benninga S., Nurko M.A., Faure C., Hyman P.E., James Roberts I. St, Schechter N.L. Childhood Functional Gastrointestinal Disodrders: Neonate/Toddler. Gastroenterology. 2016;150(6):1443-1455. https://doi. org/10.1053/j.gastro.2005.11.065.

5. Cohen-Silver J., Ratnapalan S. Management of infantile colic: a review. Clin Pediatr. 2009;48(1):14-17. https://doi.org/10.1177/0009922808323116.

6. Koletzko S., Niggemann B., Arato A., Dias J.A., Heuschkel R., Husby S. et al. European Society of Pediatric Gastroenterology, Hepatology and Nutrition. Diagnostic approach and management of cow.s-milk protein allergy in infants and children: ESPGHAN GL Committee practical guidelines. J Pediatr Gastroenterol Nutr. 2012;55(2):221-229. https://doi.org/10.1097/ MPG.0b013e31825c9482.

7. Miranda A. Early life stress and pain: an important link to functional bowel disorders. Pediatr Ann. 2009;38(5):279-282. Available at: https://pubmed.ncbi.nlm.nih.gov/19476301.

8. Shergill-Bonner R. Infantile colic: practicalities of management, including dietary aspects. J Fam Health Care. 2010;20(6):206-209. Available at: https://pubmed.ncbi.nlm.nih.gov/21319674.

9. Critch J. Infantile colic: is there a role for dietary interventions? Pediatr Child Health. 2011;16(1):47-49. Available at: https://pubmed.ncbi.nlm.nih. gov/22211076.

10. St James-Roberts I., Alvarez M., Hovish K. Emergence of a developmental explanation for prolonged crying in 1-to 4-month-old infants: review of the evidence. J Pediatr Gastroenterol Nutr. 2013;57(1):30-36. https://doi.org/10.1097/01.mpg.0000441932.07469.1b.

11. Brand S., Furlano R., Sidler M., Schulz J.C., Holsboer-Trachsler E.A. “Oh, baby, please don't cry!": in infants suffering from infantile colic hypothalamic-pituitary-adrenocortical axis activity is related to poor sleep and increased crying intensity. Neuropsychobiology. 2011;64:15-23. https://doi.org/10.1159/000322456.

12. Burns AJ., Thapar N. Developmental and postnatal changes in the enteric nervous system. J Pediatr Gastroenterol Nutr. 2013;57(1):4-8. https://doi. org/10.1097/01.mpg.0000441925.75189.01.

13. van der Wal M.F., van E.M., Bonsel G.J. Stress and emotionalproblems during pregnancy and excessive infant crying. J Dev Behav Pediatr. 2007;28(6):431-437. https://doi.org/10.1097/DBP.0b013e31811ff8f4.

14. Vik T., Grote V., Escribano J., Socha J., Verduci E., Fritsch M. et al. Infantile colic, prolonged crying and maternal postnatal depression. Acta Paediatr. 2009;98(8):1344-1348. https://doi.org/10.1111/j.1651-2227.2009.01317.x.

15. Ha-Vinh Leuchter R., Darque A., Huppi P.S. Brain maturation, early sensory processing and infant colic. J Pediatr Gastroenterol Nutr. 2013;57(1):18-25. Available at: https://journals.lww.com/jpgn/Fulltext/2013/12001/Brain_ Maturation,_Early_Sensory_Processing,_and.8.aspx.

16. Cohen E.A., Hadash A., Shehadeh N., Pillar G. Breastfeeding may improve nocturnal sleep and reduce infantile colic: potential role of breast milk melatonin Eur J Pediatr. 2012;171:729-732. https://doi.org/10.1007/s00431-011-1659-3.

17. Grenham S., Clarke G., Cryan J.F., Dinan T.G. Brain-gut-microbe communication in health and disease. Front Physiol. 2011;2:94. https://doi.org/10.3389/ fphys.2011.00094.
18. Pärtty A., Kalliomäki M., Endo A., Salminen S., Isolauri E. Compositional Development of Bifidobacterium and Lactobacillus Microbiota Is Linked with Crying and Fussing in Early Infancy. PLoS ONE. 2012;7(3):e32495. https://doi.org/10.1371/journal.pone.0032495.

19. Indrio F., Dargenio V.N., Giordano P., Francavilla R. Preventing and Treating Colic. Adv Exp Med Biol. 2019;1125:49-56. https://doi.org/10.1007/5584_2018 315.

20. de Weerth C., Fuentes S., Puylaert P., de Vos W. Intestinal Microbiota of Infants With Colic: Development and Specific Signatures. Pediatrics. 2013;131(2):550-558. https://doi.org/10.1542/peds.2012-1449.

21. Pärtty A., Kalliomäki M., Salminen S., Isolauri E. Infantile Colic Is Associated With Low-grade Systemic Inflammation. J Pediatr Gastroenterol Nutr. 2017;64(5):691-695. https://doi.org/10.1097/MPG.0000000000001340.

22. Rhoads J.M., Fatheree N.J., Norori J., Liu Y., Lucke J., Tyson J., Ferris M. Altered fecal microflora and increased fecal calprotectin in infant colic. J Pediatr. 2009;155(6):823-828. Available at: https://pubmed.ncbi.nlm.nih. gov/19628216.

23. Loughman A., Quinn T., Nation M.L., Reichelt A., Moore R.J., Van T.T.H., Sung V., Tang M.L.K. Infant microbiota in colic: predictive associations with problem crying and subsequent child behavior. J Dev Orig Health Dis. 2021;12(2): 260-270. https://doi.org/10.1017/S2040174420000227.

24. Altman R.L., Canter J., Patrick P.A., Daley N., Butt N., Brand D. Parent educationby maternity nurses and prevention of abusive head trauma. Pediatrics. 2011;128(5):1164-1172. https://doi.org/10.1542/peds.2010-3260.

25. Bechtel K., Le K., Martin K.D., Shah N., Leventhal J., Colson E. Impact of an educational intervention on caregivers' beliefs about infant cryingand knowledge of shaken baby syndrome. Acad Pediatr. 2011;11(6):481-486. https://doi.org/10.1016/j.acap.2011.08.001.

26. Макарова Е.Г., Украинцев С.Е. Функциональные расстройства органов пищеварения у детей: отдаленные последствия и современные возможности предупреждения и коррекции. Педиатрическая фармакология. 2017;14(5):392-399. https://doi.org/10.15690/pf.v14i5.1788.

27. Wolke D., Schmid G., Schreier A., Meyer R. Crying and feeding problems in infancy and cognitive outcome in preschool children born at risk: a prospective population study. J Dev Behav Pediatr. 2009;30(3):226-238. https://doi.org/10.1097/DBP.0b013e3181a85973.

28. Romanello S., Spiri D., Marcuzzi E., Zanin A., Boizeau P., Rivier S. et al. Association between childhood migraine and history of infantile colic. JAMA. 2013;309(15):1607-1612. https://doi.org/10.1001/jama.2013.747.

29. Savino F., Castaño E., Bretto R., Brondello C., Palumeri E., Oggero R. A prospective 10-year study on children who had severe infantile colic. Acta Paediatr Suppl. 2005;94(449):129-132. https://doi. org/10.1111/j.1651-2227.2005.tb02169.x.

30. Botha E., Joronen K., Kaunonen. The consequences of having an excessively crying infant in the family: an integrative literature review. Scand J Caring Sci. 2019;33(4):779-790. https://doi.org/10.1111/scs.12702.

31. Benninga M.A., Nurko S., Faure C., Hyman P., Roberts I., Schechter N. Childhood Functional Gastrointestinal Disorders: Neonate/Toddler. Gastroenterology. 2016;150(6):1443-1455. https://doi.org/10.1053/j.gastro.2016.02.016.

32. Heine R.G. Cow's-milk allergy and lactose malabsorption in infants with colic. J Pediatr Gastroenterol Nutr. 2013;57(1):25-27. https://doi. org/10.1097/01.mpg.0000441930.13307.9b.

33. Vandenplas Y., Rudolph C.D., Di Lorenzo C., Hassall E., Liptac G., Mazur L. et al. Pediatric gastroesophageal reflux clinical practice guidelines: joint recommendations of the North American Society for of Pediatric Gastroenterology, Hepatology and Nutrition (ESPGHAN).J Pediatr Gastroenterol Nutr. 2009;49(4):498-547. https://doi.org/10.1097/MPG.0b013e3181b7f563.

34. Freedman S.B., Al-Harthy N., Thull-Freedman J. The crying infant: diagnostic testing and frequency of serious underlying disease. Pediatrics. 2009:123(3):841-848. https://doi.org/10.1542/peds.2008-0113.

35. Захарова И.Н., Яцык Г.В., Боровик Т.Э., Скворцова В.А., Звонкова Н.Г., Дмитриева Ю.А. и др. Младенческие кишечные колики: современный 
взгляд на проблему. Consilium Medicum. Педиатрия (Прил.). 2014;(4):34-41. Режим доступа: https://www.pfizerprofi.ru/sites/default/files/cm pediatriya_2014.pdf.

36. Савино Ф., Захарова И.Н. Диагностика и лечение младенческих кишечных колик: мнение экспертов ESPGHAN. Consilium Medicum. Педиатрия (Прил.). 2016;(2):10-17. Режим доступа: https://omnidoctor.ru/ upload/iblock/95a/95a55b61b413b8984888ebe5d5cde1cc.pdf.

37. Daelemans S., Peeters L., Hauser B., Vandenplas Y. Recent advances in understanding and managing infantile colic. F1000Res. 2018;7:F1000. https://doi.org/10.12688/f1000research.14940.1.

38. Руженцова Т.А. Младенческие колики: от патогенеза к лечению. РМЖ. Педиатрия. 2018;(5):82-85. Режим доступа: https:/www.rmj.ru/articles/ pediatriya/Mladencheskie koliki ot patogeneza klecheniyu.

39. Waikar Y. Infantile Colic: An Overview. J Neonatal Pediatr. 2018;4(1):1-3. https://doi.org/10.4172/2572-4983.1000153.

40. Karkhaneh M., Fraser L., Jou H., Vohra S. Effectiveness of probiotics in infantile colic: A rapid review. Paediatr Child Health. 2020;25(3):149-159. https://doi.org/10.1093/pch/pxz007.

41. Skonieczna-Żydecka K., Janda K., Kaczmarczyk M., Marlicz W., Łoniewski I., Łoniewska B. The Effect of Probiotics on Symptoms, Gut Microbiota and Inflammatory Markers in Infantile Colic: A Systematic Review, MetaAnalysis and Meta-Regression of Randomized Controlled Trials. J Clin Med. 2020:9(4):999. https://doi.org/10.3390/jcm9040999.

42. Hjern A., Lindblom K., Reuter A., Silfverdal S.A. A systematic review of prevention and treatment of infantile colic. Acta Paediatr. 2020;109(9):17331744. https://doi.org/10.1111/apa.15247.

43. Pourmirzaiee M.A., Famouri F., Moazeni W., Hassanzadeh A., Hajihashemi M. The efficacy of the prenatal administration of Lactobacillus reuteri LR92 DSM 26866 on the prevention of infantile colic: a randomized control trial. Eur J Pediatr. 2020;179(10):1619-1626. https://doi.org/10.1007/ s00431-020-03641-4

44. Ringgel-Kulra T., Goldsmith J.R., Carroli I.M., Barros S. P., Palsson O., Jobin C., Ringel Y. Lactobacillus acidophilus NCFM affects colonic mucosa opioid receptor expression in patient with functional abdominal pain a randomized clinical study. Aliment Pharmacol Ther. 2014;40(2):200-207. https://doi.org/10.1111/apt.12800.

45. Szajewska H., Konarska Z., Kołodziej M. Probiotic Bacterial and Fungal Strains: Claims with Evidence. Dig Dis. 2016;34(3):251-259. https://doi.org/10.1159/000443359.

46. Savino F., Montanari P., Galliano I., Daprà V., Bergallo M. Lactobacillus rhamnosus GG (ATCC 53103) for the Management of Infantile Colic: A Randomized Controlled Trial. Nutrients. 2020;12(6):1693. https://doi.org/10.3390/nu12061693.

47. Brenner D.M., Chey W.D. Bifidobacterium infantis: a novel probiotic for the treatment ofirritable bowel syndrome. Rev Gastroenterol Disord. 2009;9(1):7-15. Available at: https://pubmed.ncbi.nlm.nih.gov/19367213.
48. Nocerino R., De Filippis F., Cecere G., Marino A., Micillo M., Di Scala C. et al. The therapeutic efficacy of Bifidobacterium animalis subsp. lactis BB-12 ${ }^{\circledR}$ in infant colic: A randomised, double blind, placebo-controlled trial. Aliment Pharmacol Ther. 2020;51(1):110-120. https://doi.org/10.1111/apt.15561.

49. Maldonado-Lobón J.A., Blanco-Rojo R., Maldonado J., Ali M.A., Almazán M.V., Suanes-Cabello A. et al. Efficacy of Bifidobacterium breve CECT7263 for infantile colic treatment: an open-label, parallel, randomised, controlled trial. Benef Microbes. 2021;12(1):55-67. https://doi.org/10.3920/BM2020.0105.

50. Chen K., Liu C., Li H., Lei Y., Zeng C., Xu S. et al. Infantile Colic Treated With Bifidobacterium longum CECT7894 and Pediococcus pentosaceus CECT8330: A Randomized, Double-Blind, Placebo-Controlled Trial. Front Pediatr. 2021;9:635176. Available at: https://www.frontiersin.org/articles/10.3389/fped.2021.635176/full.

51. Yala E.T. The Clinical Efficacy of Multi-strain Probiotics (Bac-Set) in the Management of Acute Gastroenteritis in Children Two Months to Two Years Old. PIDSP Journal. 2010;11(2):86-91. Available at: http://pidsphil.org/pdf/Journal_12312011/jo39_ja05.pdf

52. Kianifar H., Ahanchian H., Grover Z., Jafari S., Noorbakhsh Z., Khakshour A. et al. Synbiotic in the management of infantile colic: A randomized controlled trial. J Paed Chil Heal. 2014;50(10):801-805. https://doi.org/10.1111/jpc.12640.

53. Харитонова Л.А., Кучеря Т.В. Применение пробиотика в педиатрической практике: результаты клинического исследования. Доктор.ру. 2016;(6):38-41. Режим доступа: https://journaldoctor.ru/catalog/pediatriya/primenenie-probiotika-v-pediatricheskoy-praktike-rezultatyklinicheskogo-issledovaniya/?lang=ru\&clear_cache $=Y$.

54. Hojsak I. Probiotics in Functional Gastrointestinal Disorders Adv Exp Med Biol. 2019;1125:121-137. https://doi.org/10.1007/5584 2018321.

55. Ishaque M., Khosruzzaman S., Ahmed D., Sah М. Рандомизированное плацебоконтролируемое клиническое исследование мультипробиотика Bio-Kult ${ }^{\circledR}$ при лечении синдрома раздраженного кишечника с преобладанием диареи. Медицинский совет. 2018;(14):98-108. https://doi.org/10.21518/2079-701Х-2018-14-98-108.

56. Захарова И.Н., Ардатская М.Д., Сугян Н.Г. Влияние мультиштаммового пробиотика на метаболическую активность кишечной микрофлоры у детей грудного возраста с функциональными нарушениями желудочно-кишечного тракта: результаты плацебо-контролируемого исследования. Вопросы современной педиатрии. 2016;15(1):68-73. https://doi.org/10.15690/vsp.v15i1.1501.

57. Захарова И.Н., Сугян Н.Г. Мультипробиотик Бак-Сет. Результаты российских и зарубежных клинических исследований. Медицинский совет. 2017;(19):104-110. https://doi.org/10.21518/2079-701X-2017-19-104-110.

58. Simonson J., Haglund K., Weber E., Fial A., Hanson L. Probiotics for the Management of Infantile Colic: A Systematic Review. MCN Am J Matern Child Nurs. 2021;46(2):88-96. https://doi.org/10.1097/NMC.0000000000000691.

\section{References}

1. Belyaeva I.A., Namazova-Baranova L.S., Potekhina T.V. Infantile colic: a new look at an old problem. Pediatria. 2015;94(1):137-144. (In Russ.) Available at: https://pediatriajournal.ru/files/upload/mags/344/2015_14212.pdf.

2. Wessel M.A., Cobb J.C., Jackson E.B., Harris G.S. Jr., Detwiler A.C. Paroxysmal fussing in infancy, sometimes called colic. Pediatrics. 1954:14(5):421-435. Available at: https://pubmed.ncbi.nlm.nih.gov/13214956.

3. Vandenplas Y., Gutierrez-Castrellon P., Velasco-Benitez C., Palacios J., Jaen D., Ribeiro H. et al. Practical algorithms for managing common gastrointestinal symptoms in infants. Nutrition. 2013;29(1):184-194. https://doi. org/10.1016/j.nut.2012.08.008

4. Benninga S., Nurko M.A., Faure C., Hyman P.E., James Roberts I. St, Schechter N.L. Childhood Functional Gastrointestinal Disodrders: Neonate/Toddler. Gastroenterology. 2016;150(6):1443-1455. https://doi. org/10.1053/j.gastro.2005.11.065.

5. Cohen-Silver J., Ratnapalan S. Management of infantile colic: a review. Clin Pediatr. 2009;48(1):14-17. https://doi.org/10.1177/0009922808323116.

6. Koletzko S., Niggemann B., Arato A., Dias J.A., Heuschkel R., Husby S. et al. European Society of Pediatric Gastroenterology, Hepatology and Nutrition. Diagnostic approach and management of cow.s-milk protein allergy in infants and children: ESPGHAN GL Committee practical guidelines. J Pediatr Gastroenterol Nutr. 2012;55(2):221-229. https://doi.org/10.1097/ MPG.0b013e31825c9482.

7. Miranda A. Early life stress and pain: an important link to functional bowel disorders. Pediatr Ann. 2009;38(5):279-282. Available at: https://pubmed.ncbi.nlm.nih.gov/19476301.

8. Shergill-Bonner R. Infantile colic: practicalities of management, including dietary aspects. J Fam Health Care. 2010;20(6):206-209. Available at: https://pubmed.ncbi.nlm.nih.gov/21319674.

9. Critch J. Infantile colic: is there a role for dietary interventions? Pediatr Child Health. 2011;16(1):47-49. Available at: https://pubmed.ncbi.nlm.nih.gov/22211076

10. St James-Roberts I., Alvarez M., Hovish K. Emergence of a developmental explanation for prolonged crying in 1-to 4-month-old infants: review of the evidence. J Pediatr Gastroenterol Nutr. 2013;57(1):30-36. https://doi. org/10.1097/01.mpg.0000441932.07469.1b.
11. Brand S., Furlano R., Sidler M., Schulz J.C., Holsboer-Trachsler E.A. “Oh, baby, please don't cry!": in infants suffering from infantile colic hypothalamic-pituitary-adrenocortical axis activity is related to poor sleep and increased crying intensity. Neuropsychobiology. 2011;64:15-23. https://doi.org/10.1159/000322456

12. Burns A.J., Thapar N. Developmental and postnatal changes in the enteric nervous system. J Pediatr Gastroenterol Nutr. 2013;57(1):4-8. https://doi. org/10.1097/01.mpg.0000441925.75189.01

13. van der Wal M.F., van E.M., Bonsel G.J. Stress and emotionalproblems during pregnancy and excessive infant crying. J Dev Behav Pediatr. 2007;28(6):431-437. https://doi.org/10.1097/DBP.0b013e31811ff8f4.

14. Vik T., Grote V., Escribano J., Socha J., Verduci E., Fritsch M. et al. Infantile colic, prolonged crying and maternal postnatal depression. Acta Paediatr. 2009;98(8):1344-1348. https://doi.org/10.1111/j.1651-2227.2009.01317.x.

15. Ha-Vinh Leuchter R., Darque A., Huppi P.S. Brain maturation, early sensory processing and infant colic. J Pediatr Gastroenterol Nutr. 2013;57(1):18-25. Available at: https://journals.lww.com/jpgn/Fulltext/2013/12001/Brain Maturation,_Early_Sensory_Processing,_and.8.aspx.

16. Cohen E.A., Hadash A., Shehadeh N., Pillar G. Breastfeeding may improve nocturnal sleep and reduce infantile colic: potential role of breast milk melatonin. Eur J Pediatr. 2012;171:729-732. https://doi.org/10.1007/s00431-011-1659-3.

17. Grenham S., Clarke G., Cryan J.F., Dinan T.G. Brain-gut-microbe communication in health and disease. Front Physiol. 2011;2:94. https://doi.org/10.3389/ fphys.2011.00094.

18. Pärtty A., Kalliomäki M., Endo A., Salminen S., Isolauri E. Compositional Development of Bifidobacterium and Lactobacillus Microbiota Is Linked with Crying and Fussing in Early Infancy. PLoS ONE. 2012;7(3):e32495. https://doi.org/10.1371/journal.pone.0032495.

19. Indrio F., Dargenio V.N., Giordano P., Francavilla R. Preventing and Treating Colic. Adv Exp Med Biol. 2019;1125:49-56. https://doi.org/10.1007/5584_2018_315.

20. de Weerth C., Fuentes S., Puylaert P., de Vos W. Intestinal Microbiota of Infants With Colic: Development and Specific Signatures. Pediatrics. 2013;131(2):550-558. https://doi.org/10.1542/peds.2012-1449. 
21. Pärtty A., Kalliomäki M., Salminen S., Isolauri E. Infantile Colic Is Associated With Low-grade Systemic Inflammation. J Pediatr Gastroenterol Nutr. 2017;64(5):691-695. https://doi.org/10.1097/MPG.0000000000001340.

22. Rhoads J.M., Fatheree N.J., Norori J., Liu Y., Lucke J., Tyson J., Ferris M. Altered fecal microflora and increased fecal calprotectin in infant colic. J Pediatr. 2009;155(6):823-828. Available at: https://pubmed.ncbi.nlm.nih gov/19628216.

23. Loughman A., Quinn T., Nation M.L., Reichelt A., Moore R.J., Van T.T.H., Sung V., Tang M.L.K. Infant microbiota in colic: predictive associations with problem crying and subsequent child behavior. J Dev Orig Health Dis. 2021;12(2): 260-270. https://doi.org/10.1017/S2040174420000227.

24. Altman R.L., Canter J., Patrick P.A., Daley N., Butt N., Brand D. Parent educationby maternity nurses and prevention of abusive head trauma. Pediatrics. 2011;128(5):1164-1172. https://doi.org/10.1542/peds.2010-3260.

25. Bechtel K., Le K., Martin K.D., Shah N., Leventhal J., Colson E. Impact of an educational intervention on caregivers' beliefs about infant cryingand knowledge of shaken baby syndrome. Acad Pediatr. 2011;11(6):481-486. https://doi.org/10.1016/j.acap.2011.08.001.

26. Makarova E.G., Ukraintsev S.E. Functional Gastrointestinal Disorders in Infants: Long-Tern Consequences and Modern Approaches for Prevention and Treatment. Pediatricheskaya farmakologiya = Pediatric Pharmacology. 2017;14(5):392-399. (In Russ.) https://doi.org/10.15690/pf.v14i5.1788.

27. Wolke D., Schmid G., Schreier A., Meyer R. Crying and feeding problems in infancy and cognitive outcome in preschool children born at risk: a prospective population study. J Dev Behav Pediatr. 2009;30(3):226-238. https://doi.org/10.1097/DBP.0b013e3181a85973.

28. Romanello S., Spiri D., Marcuzzi E., Zanin A., Boizeau P., Rivier S. et al. Association between childhood migraine and history of infantile colic. JAMA. 2013;309(15):1607-1612. https://doi.org/10.1001/jama.2013.747.

29. Savino F., Castaño E., Bretto R., Brondello C., Palumeri E., Oggero R. A prospec tive 10-year study on children who had severe infantile colic. Acta Paediatr Suppl. 2005;94(449):129-132. https://doi.org/10.1111/j.1651-2227.2005. tb02169.x.

30. Botha E., Joronen K., Kaunonen. The consequences of having an excessively crying infant in the family: an integrative literature review. Scand J Caring Sci. 2019;33(4):779-790. https://doi.org/10.1111/scs.12702.

31. Benninga M.A., Nurko S., Faure C., Hyman P., Roberts I., Schechter N. Childhood Functional Gastrointestinal Disorders: Neonate/Toddler. Gastroenterology. 2016;150(6):1443-1455. https://doi.org/10.1053/j.gastro.2016.02.016

32. Heine R.G. Cow's-milk allergy and lactose malabsorption in infants with colic. J Pediatr Gastroenterol Nutr. 2013;57(1):25-27. https://doi. org/10.1097/01.mpg.0000441930.13307.9b.

33. Vandenplas Y., Rudolph C.D., Di Lorenzo C., Hassall E., Liptac G., Mazur L. et al. Pediatric gastroesophageal reflux clinical practice guidelines: joint recommendations of the North American Society for of Pediatric Gastroenterology, Hepatology and Nutrition (ESPGHAN). J Pediatr Gastroenterol Nutr. 2009:49(4):498-547. https://doi.org/10.1097/MPG.0b013e3181b7f563.

34. Freedman S.B., Al-Harthy N., Thull-Freedman J. The crying infant: diagnostic testing and frequency of serious underlying disease. Pediatrics. 2009;123(3):841-848. https://doi.org/10.1542/peds.2008-0113.

35. Zakharova I.N., Yatsyk G.V., Borovik T.E., Skvortsova V.A., Zvonkova N.G., Dmitrieva Yu.A. et al. Infantile intestinal colic: modern view on the problem. Consilium Medicum. Pediatrics (Suppl.). 2014;(4):34-41. (In Russ.) Available at: https://www.pfizerprofi.ru/sites/default/files/cm pediatriya_2014.pdf.

36. Savino F., Zakharova I.N. Diagnosis and treatment of infantile colic: the experts ESPGHAN opinion. Consilium Medicum. Pediatrics (Suppl.). 2016;(2):10-17. (In Russ.) Available at: https://omnidoctor.ru/upload/iblock /95a/95a55b61b413b8984888ebe5d5cde1cc.pdf.

37. Daelemans S., Peeters L., Hauser B., Vandenplas Y. Recent advances in understanding and managing infantile colic. F1000Res. 2018;7:F1000 https://doi.org/10.12688/f1000research.14940.1.

38. Ruzhentsova T.A. Baby colics: from pathogenesis to treatment. $R M Z h=R M J .2018 ;(5): 82-85$. (In Russ.) Available at: https://www.rmj.ru/ articles/pediatriya/Mladencheskie koliki ot patogeneza klecheniyu

39. Waikar Y. Infantile Colic: An Overview. J Neonatal Pediatr. 2018;4(1):1 - 3 . https://doi.org/10.4172/2572-4983.1000153.

40. Karkhaneh M., Fraser L., Jou H., Vohra S. Effectiveness of probiotics in infantile colic: A rapid review. Paediatr Child Health. 2020;25(3):149-159. https://doi.org/10.1093/pch/pxz007.

41. Skonieczna-Żydecka K., Janda K., Kaczmarczyk M., Marlicz W., Łoniewski I., Łoniewska B. The Effect of Probiotics on Symptoms, Gut Microbiota and
Inflammatory Markers in Infantile Colic: A Systematic Review, MetaAnalysis and Meta-Regression of Randomized Controlled Trials. J Clin Med. 2020;9(4):999. https://doi.org/10.3390/jcm9040999.

42. Hjern A., Lindblom K., Reuter A., Silfverdal S.A. A systematic review of prevention and treatment of infantile colic. Acta Paediatr. 2020;109(9):1733-1744. https://doi.org/10.1111/apa.15247.

43. Pourmirzaiee M.A., Famouri F., Moazeni W., Hassanzadeh A., Hajihashemi M. The efficacy of the prenatal administration of Lactobacillus reuteri LR92 DSM 26866 on the prevention of infantile colic: a randomized control trial. Eur J Pediatr. 2020;179(10):1619-1626. https://doi.org/10.1007/ s00431-020-03641-4.

44. Ringgel-Kulra T., Goldsmith J.R., Carroli I.M., Barros S. P., Palsson O., Jobin C., Ringel Y. Lactobacillus acidophilus NCFM affects colonic mucosal opioid receptor expression in patient with functional abdominal pain a randomized clinical study. Aliment Pharmacol Ther. 2014;40(2):200-207. https://doi.org/10.1111/apt.12800.

45. Szajewska H., Konarska Z., Kołodziej M. Probiotic Bacterial and Fungal Strains: Claims with Evidence. Dig Dis. 2016;34(3):251-259. https://doi.org/10.1159/000443359.

46. Savino F., Montanari P., Galliano I., Daprà V., Bergallo M. Lactobacillus rhamnosus GG (ATCC 53103) for the Management of Infantile Colic: A Randomized Controlled Trial. Nutrients. 2020;12(6):1693. https://doi.org/10.3390/nu12061693.

47. Brenner D.M., Chey W.D. Bifidobacterium infantis: a novel probiotic for the treatment ofirritable bowel syndrome. Rev Gastroenterol Disord. 2009;9(1):7-15. Available at: https://pubmed.ncbi.nlm.nih.gov/19367213.

48. Nocerino R., De Filippis F., Cecere G., Marino A., Micillo M., Di Scala C. et al. The therapeutic efficacy of Bifidobacterium animalis subsp. lactis BB-12 ${ }^{\circledR}$ in infant colic: A randomised, double blind, placebo-controlled trial. Aliment Pharmacol Ther. 2020;51(1):110-120. https://doi.org/10.1111/apt.15561.

49. Maldonado-Lobón J.A., Blanco-Rojo R., Maldonado J.,Ali M.A.,Almazán M.V., Suanes-Cabello A. et al. Efficacy of Bifidobacterium breve CECT7263 for infantile colic treatment: an open-label, parallel, randomised, controlled trial. Benef Microbes. 2021;12(1):55-67. https://doi.org/10.3920/BM2020.0105.

50. Chen K., Liu C., Li H., Lei Y., Zeng C., Xu S. et al. Infantile Colic Treated With Bifidobacterium longum CECT7894 and Pediococcus pentosaceus CECT8330: A Randomized, Double-Blind, Placebo-Controlled Trial. Front Pediatr. 2021;9:635176. Available at: https://www.frontiersin.org/ articles/10.3389/fped.2021.635176/full.

51. Yala E.T. The Clinical Efficacy of Multi-strain Probiotics (Bac-Set) in the Management of Acute Gastroenteritis in Children Two Months to Two Years Old. PIDSP Journal. 2010;11(2):86-91. Available at: http://pidsphil.org/pdf/Journal_12312011/jo39_ja05.pdf.

52. Kianifar H., Ahanchian H., Grover Z., Jafari S., Noorbakhsh Z., Khakshour A. et al. Synbiotic in the management of infantile colic: A randomized controlled trial. J Paed Chil Heal. 2014;50(10):801-805. https://doi.org/10.1111/ jpc. 12640 .

53. Kharitonova L.A., Kucherya T.V. Using Probiotics in Pediatric Practice: Clinical Study Results. Doktor.Ru. 2016;(6):38-41. (In Russ.) Available at: https://journaldoctor.ru/catalog/pediatriya/primenenie-probiotika-vpediatricheskoy-praktike-rezultaty-klinicheskogoissledovaniya/?lang=ru\& clear cache $=Y$.

54. Hojsak I. Probiotics in Functional Gastrointestinal Disorders Adv Exp Med Biol. 2019;1125:121-137. https://doi.org/10.1007/5584_2018_321.

55. Ishaque M., Khosruzzaman S., Ahmed D., Sah M. A randōmized placebocontrolled clinical trial of a multi-strain probiotic formulation (Bio-Kult ${ }^{\circledR}$ ) in the management of diarrheapredominant irritable bowel syndrome. Meditsinskiy sovet = Medical Council. 2018;(14):98-108. (In Russ.) https://doi.org/10.21518/2079-701X-2018-14-98-108.

56. Zakharova I.N., Ardatskaya M.D., Sugyan N.G. The Effect of MultiStrain Probiotic on the Metabolic Activity of the Intestinal Microflora in Infants with Functional Disorders of the Gastrointestinal Tract: the Results of a Placebo-Controlled Study. Voprosy sovremennoj pediatrii = Current Pediatrics. 2016;15(1):68-73. (In Russ.) https://doi. org/10.15690/vsp.v15i1.1501.

57. Zakharova I.N., Sugyan N.G. Bac-set multiprobiotic: results of russian and foreign clinical research. Meditsinskiy sovet = Medical Council. 2017;(19):104110. (In Russ.) https://doi.org/10.21518/2079-701X-2017-19-104-110.

58. Simonson J., Haglund K., Weber E., Fial A., Hanson L. Probiotics for the Management of Infantile Colic: A Systematic Review. MCN Am J Matern Child Nurs. 2021;46(2):88-96. https://doi.org/10.1097/ NMC.0000000000000691.

\section{Информация об авторе:}

Панова Людмила Дмитриевна, д.м.н., доцент, профессор кафедры госпитальной педиатрии, Башкирский государственный медицинский университет; 450008, Россия, Уфа, ул. Ленина, д. 3; panov_home@ufacom.ru

\section{Information about the author:}

Luydmila D. Panova, Dr. Sci. (Med.), Assistant Professor, Professor of the Department of Hospital Pediatrics, Bashkir State Medical University; 3, Lenin St., Ufa, 450008, Russia; panov_home@ufacom.ru 